\title{
Application of the quartz crystal microbalance technique in corrosion studies. A review ${ }^{1}$
}

\author{
M.A. Petrunin, N.A. Gladkikh, M.A. Maleeva, T.A. Yurasova, \\ E.V. Terekhova and L.B. Maksaeva*
}

A.N. Frumkin Institute of Physical Chemistry and Electrochemistry, Russian Academy of Sciences, Leninsky pr. 31, 119071 Moscow, Russian Federation

*E-mail: Imaksaeva@mail.ru

\begin{abstract}
Various fields of application of the quartz crystal microbalance technique (QCM) and its modification, electrochemical quartz crystal nanobalance technique (EQCN), in studies on surface phenomena occurring during metal corrosion are discussed. The theory of the method, design concepts of a quartz resonator, piezoelectric sensor, and experimental setups are outlined. Factors affecting the frequency of oscillations of a quartz resonator, QCM and EQCN studies of the interaction of gases, water vapor, and various organic compounds with thin metal films are characterized. The processes of atmospheric corrosion, determination of the mass of aerosols precipitating from air and of the component composition of the atmosphere are described in detail. The prospects of using QCM and EQCN in studying the regularities of corrosion processes in electrolytes, adsorption of corrosion inhibitors and organosilicon monomers on the metal surface from gas and liquid media are discussed together with the mechanisms of growth and dissolution of a passive film on metals. Examples of application of the QCM method in combination with other physicochemical methods, such as the measurement of the electronic work function, contact potential difference, Auger spectroscopy, electron and atomic force microscopy, X-ray photoelectron spectroscopy, IR spectroscopy, and a number of electrochemical methods, are provided.
\end{abstract}

Keywords: piezoelectricity, quartz crystal nanobalance, quartz resonator, metaloxidation, metal corrosion, adsorption, corrosion inhibitors, electrochemical quartz crystal microbalance, atmospheric corrosion, oxide films on metals.

Received: October 31, 2019. Published: January 29, 2020

doi: $\underline{10.17675 / 2305-6894-2020-9-1-6}$

\footnotetext{
${ }^{1}$ This study was supported by the Basic Research Program of the Presidium of the Russian Academy of Sciences No. 34P "Urgent Problems of Surface Physicochemistry and Creation of New Composite Materials. 4. Nanostructured coatings for electronics, photonics, alternative energy sources and materials protection" and partially supported by the Russian Foundation for basic research, project No. 17-03-00232.
} 


\section{Introduction}

Piezoelectric devices and equipment play an important role in radioelectronic industry. Piezoelectric devices in particular occupy a prominent place. Quartz crystal resonators are way ahead of other types of electromechanical resonance converters as regards the whole set of key indicators (working frequency range, Q-factor, time and temperature stability, reproducibility of parameters, dimensions, weight, cost) [1].

The production of quartz crystal resonators is characterized by a high technology level and is based on using batch production methods typical of microelectronics, including industrial growth of quartz crystals. Production of piezoelectric devices is constantly expanding. The annual volume of $\alpha$-quartz production was 3000 tons in 1997 and 2 bln units in 2010, to the total amount of 4.5 bln dollars per year [2].

Starting from the second half of the twentieth century, the scope of application of resonance piezodevices keeps expanding. While the main sphere of application of quartz crystal resonators in the first half of this century involved radiotechnical systems and communication devices, later the market of these devices was basically determined by the demands of the clock industry, microprocessor technology, and a wide spectrum of radiotechnical devices.

The first attempts of using piezoresonators in devices for the measurement of physical values were made in the late forties of the twentieth century, though a wide application of resonators in quartz crystal clocks started back in 1928 [3]. Widespread application of sensors based on controlled quartz crystal resonators did not start until the early 1960s. It is at this time that significant progress was achieved in the development of piezoelectric devices for frequency stabilization, digital frequency measurement techniques were introduced, and commercial artificial growing of quartz was started.

In 1959, application of quartz resonators for precision weighing (quartz crystal microand nanobalance) was first suggested [4]: a high-sensitivity method for determining the weight of substances applied (deposited) onto a surface with the accuracy better than an atomic monolayer $[5,6]$.

The second half of the twentieth century was characterized by a rampant development of innovative methods for studying the physical and chemical properties of materials, such as Auger [7] and X-ray photoelectron spectroscopy (XPS) [8-11], scanning electron microscopy [12], X-ray microanalysis [13], ionic and atomic emission spectroscopy [14], Fourier transform IR spectroscopy [15-17], secondary ion mass spectrometry [18, 19], low energy electron diffraction [20], etc. Owing to this, the options for studying surface phenomena related to adsorption, oxidation and metal corrosion expanded considerably. Nevertheless, the weighing methods that allow determining the mass variation of surface substances still occupy an important place in research in physics and chemistry. Owing to rapid development of various fields of science and technology related to the synthesis and application of thin films, numerous studies of processes causing insignificant mass 
variation, namely variations of about micrograms or nanograms [21], use methods that allow measuring such mass variations.

The most significant advances in the field of microbalance starting from the sixties of the twentieth century are reflected in the proceedings of the "Vacuum Microbalance Techniques" conferences [22-24]. Despite its long-term application, the method of quartz crystal microbalance (QCM) continues to attract the attention of researchers owing to its relative design simplicity. Thus, already in the late twentieth century, this provided means for measuring masses of about $10^{-7}$ to $10^{-8} \mathrm{~g} / \mathrm{cm}^{2}$ and in some cases $10^{-10}$ to $10^{-12} \mathrm{~g} / \mathrm{cm}^{2}$ [21]. The QCM method is practically inertialess (the response is about several microseconds) $[2,21,25,26]$. Crystal frequency variations caused by factors other than mass variation can be minimized or corrected. Besides, the weighing results obtained using the QCM technique are not affected by mechanical impacts or vibrations, as opposed to beam, torsion, spring and other types of balances with mechanical hangers [25].

These advantages led to extensive use of QCM in studies of atmospheric corrosion, adsorption of gas components and corrosion inhibitors, metal oxidation, formation and mechanism of protective properties of ultrathin layers and films, kinetics of heterogeneous chemical reactions, and sensitive detection in gas chromatography [21]. QCM is used to solve the problems of water content measurements in air, determination of aerosol concentrations and composition of corrosive atmospheric components, measurements of surface diffusion, vapor pressure of solids and liquids, measurement and control of the thickness of sputtered (vapor-deposited) metal films, measurement of adsorption, "true" surface area, and roughness coefficient [21, 26-30].

After development of the QCM technique in liquid media suggested in early eighties of the twentieth century $[5,31,32]$ and used in combination with electrochemical quartz crystal nanobalance (EQCN) [5], the scope of QCM application expanded considerably. It included determination of the rate of deposition of galvanic coatings from electrolytes $[5,6,21,31]$, studies of atmospheric corrosion processes $[6,21,27]$ and corrosion in electrolytes [6, 26, 33-35], behavior of corrosion inhibitors [5, 6, 28, 30,33-36], as well as growth and dissolution of passive films on metals [5, 37].

Thus, apart from radioelectronics, quartz crystal resonators are widely used in research work for monitoring the mass variation of the surfaces of solids. Comprehensive reviews on the application of various microbalance types in studies of surface phenomena on solids are provided in [5, 21, 26, 38]. Besides, issues related to the QCM technique are discussed in detail in $[2,6,22-24,34,39,40]$. The application of the QCM method in studies of corrosion phenomena is not described in sufficient detail in monographs and reviews. In view of this, the present work attempts to summarize the experience accumulated by Russian and foreign researchers in using QCM in the studies on corrosion and metal protection. 


\section{Theory of the QCM method}

The QCM method is based on the ability of a quartz plate (quartz resonator) in a certain vibration mode to change its resonance frequency when some foreign material is applied onto it or removed from it. This effect has been studied in detail and compared with a torsion balance in [4]. Owing to the inverse piezoeffect characteristic of quartz [1] exposed to an AC electric field, through-thickness shear modes appear in the quartz plate (standing ultrasonic waves represent a superposition of two waves moving in the plate in opposite directions).

For shear modes, the basic resonance frequency and the plate thickness are related through relationship (1) $[5,21]$ :

$$
f_{0}=\frac{N}{\tau}
$$

where $f_{0}$ is the main resonance frequency $(\mathrm{Hz}), \tau$ is the plate thickness $(\mathrm{mm}), N$ is the frequency constant of quartz depending on the orientation of the cut plate with respect to the basic quartz single crystal crystallographic axes, i.e., the cutoff angle [1].

For AT-cut crystals $\left(35^{\circ} 14^{\prime}\right)$ with a low temperature coefficient of frequency (TCF), $N=1670 \mathrm{kHz} \cdot \mathrm{mm}$; for a BT-cut $\left(-49^{\circ}\right), T=2500 \mathrm{kHz} \cdot \mathrm{mm}[1,21,26]$.

Differentiation of equation (1) by the plate thickness and substitution of the quartz plate thickness increment found through mass (2),

$$
d \tau=\frac{1}{\rho S} d m
$$

yields the resonance frequency variation as a function on the mass deposited onto the quartz plate [21]:

$$
d f=-\frac{f_{0}^{2}}{N \rho S} d m
$$

where $d \tau$ is the quartz plate thickness increment, $d f$ is the resonance frequency variation $(\mathrm{Hz}), \rho$ is the density of quartz, $2.65 \mathrm{~g} / \mathrm{cm}^{3}[1,21,26], d m$ is the mass of the material deposited on the quartz plate $(\mathrm{g}), S$ is the area of the quartz plate $\left(\mathrm{cm}^{2}\right)$ usually assumed equal to the overall area of the exciting electrodes on the plate surface, as the vibrations in the plate outside the electrodes are negligibly small and the presence of a foreign substance in these regions contributes about $1 \%$ to the overall frequency variation [4, 21].

The correctness of relationship (3) was repeatedly tested using other weighing techniques [25]. Besides, equation (3) was derived independently based on the theory of propagation of vibrations in a highly elastic liquid medium $[5,6,21]$.

AT-cut quartz resonators with the lowest TCF are most often used for weighing. For example, the TCF for an AT-cut $\left(35^{\circ} 20^{\prime}\right)$ is $0\left({ }^{\circ} \mathrm{C}\right)^{-1}$ at $30^{\circ} \mathrm{C}$ and is $< \pm 5 \cdot 10^{-6}\left({ }^{\circ} \mathrm{C}\right)^{-1}$ in the range of $\pm 30^{\circ} \mathrm{C}[1,21]$. Quartz thermostating is also used to decrease the effect of temperature on the mass measurement accuracy [26]. 
The sensitivity of the QCM method is expressed by the following relationship:

$$
\frac{f_{0}^{2}}{N \rho}=C_{f_{0}}
$$

where $C_{f_{0}}$ is the sensitivity of mass determination.

The mass is calculated from the known quartz plate frequency [4].The experimentally measured sensitivity value is very high and practically coincides with the value calculated by equation (4) $[6,21]$. In fact, when quartz with an initial resonance frequency of $10 \mathrm{MHz}$ is used, a frequency variation of $1 \mathrm{~Hz}$ corresponds to the mass variation of $4.5 \mathrm{ng} / \mathrm{cm}^{2}$, which is equivalent to the mass of $4 \%$ of a metallic iron monolayer [5].

A linear dependence of resonance frequency variation on the amount of material deposited on a quartz plate is preserved only until the thickness of the applied substance layer is substantially smaller than the quartz layer thickness. The maximum resonance frequency variation must not exceed $0.5 \%$ of the basic resonance frequency if the acceptable deviation from linearity is $1 \%$.The empirically found value of the maximum resonance frequency variation for quartz plates with $5 \mathrm{MHz}$ resonance frequency is in the range of $5-100 \mathrm{kHz}$, which corresponds to $1-2 \mathrm{mg} / \mathrm{cm}^{2}$ of a substance deposited on quartz [26]. The corresponding thickness of a layer with mass $M, d=\Delta M \cdot 10^{-8} \rho S(\AA)$, changes from 5000 to $50000 \AA$ for substances with various densities of deposited films.

A working quartz oscillator can be described by an equivalent electric circuit (Figure 1).

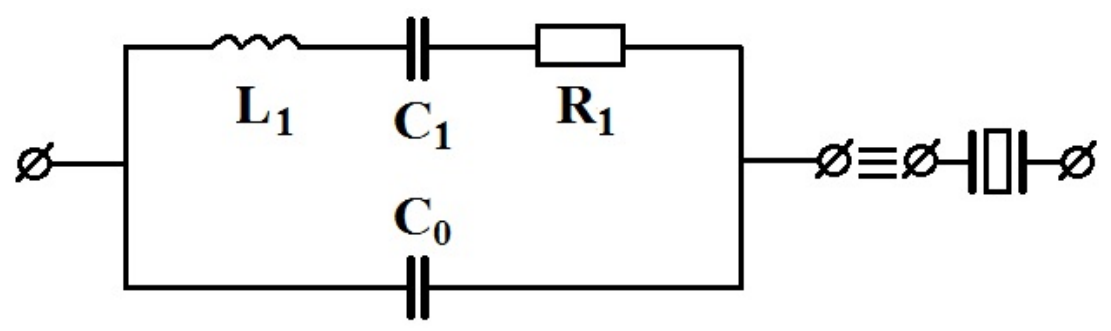

Figure 1. Equivalent circuit of a quartz resonator. $L_{1}, C_{1}, R_{1}$ are the equivalent inductance, capacitance, and loss resistance, respectively; $C_{0}$ is the static capacitance.

The electric circuit consists of two parallel branches: one of them is the so called flexible bond consisting or $R_{1}, L_{1}$, and $C_{1}$ connected in series. It is a scheme that simulates a vibrating element. The other is the so called static branch formed by capacitor $C_{0}$ related to electric capacitance arising in a dielectric material placed between two electrodes, or a static inter-electrode capacitance [1].

The resonator impedance can be expressed by the formula [42]:

$$
Z_{k}=\frac{R_{1}(1+j \alpha)}{1-\alpha \delta_{0}+j \delta_{0}}
$$


where $\alpha=2 \frac{\Delta f}{f_{s}} Q$ is the generalized resonator mistuning; $\Delta f / f_{s}=\left(f-f_{s}\right) / f_{s} ; f_{s}$ is the serial resonator frequency; $f$ is the resonator operating frequency; $Q=\sqrt{\frac{L_{1}}{C_{1}}} \cdot \frac{1}{R_{1}}$ is the resonator $Q$-factor; and $\delta_{0}=2 \pi f K C_{0} R_{1}$ is the ratio of the loss resistance to the reactive resistance of a static capacitance.

The equivalent circuit (Figure 1) can be transformed into a serial equivalent circuit (Figure 2) [42].

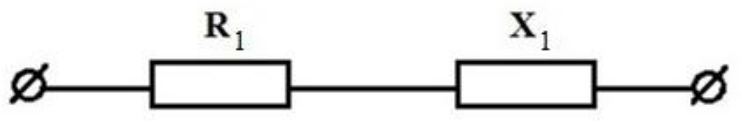

Figure 2. Serial equivalent circuit of a quartz resonator.

The equivalent serial reactive and active components can be expressed as follows [42]:

$$
\begin{gathered}
R_{k}=\frac{R_{1}}{\left(1-\alpha \delta_{0}\right)^{2}+\delta_{0}^{2}} \\
X_{\alpha}=R_{1} \cdot \frac{\alpha\left(1-\alpha \delta_{0}\right)-\delta_{0}}{\left(1-\alpha \delta_{0}\right)^{2}+\delta_{0}^{2}}
\end{gathered}
$$

Phase angle $\varphi_{\mathrm{k}}$ and the slope of the phase angle of a quartz resonator resistance as functions of the mistuning are ((8), (9)) [42]:

$$
\begin{gathered}
\operatorname{tg} \varphi_{k}=\alpha\left(1-\alpha \delta_{0}\right)-\delta_{0} \\
\frac{d \varphi_{k}}{d \alpha}=\frac{1-2 \alpha \delta_{0}}{1+\left[\alpha\left(1-\alpha \delta_{0}\right)-\delta_{0}\right]^{2}}
\end{gathered}
$$

where $d \varphi_{k} / d \alpha$ is the slope of the phase angle of the quartz resonator.

The following conclusions can be made from these equations. The dependences of $X_{k}$ and $R_{k}$ on the frequency are two-valued. For example, the same $X_{k}$ value can be observed at two frequencies, i.e., two frequencies can be excited at the same value of the circuit resonant frequency. For oscillatory schemes, the operating mode near the parallel resonance is unstable and self-oscillations are impossible [1,42]. An increase in the mistuning causes an increase in active resistance $R_{k}$, so that the conditions of selfexcitation for serial resonance generators deteriorate.

With an increase in $\delta_{0}$, the inductive resistance maximum of quartz decreases; at $\delta_{0} \geq 0.5$, a quartz resonator is equivalent to a capacitor. In this case, excitation of quartz 
resonators is impossible in oscillatory schemes. An increase in $\alpha$ and $\delta_{0}$ leads to a decrease in the slope of the phase of the quartz resonator resistance. Here, the stabilizing ability of the quartz resonator decreases [42].

To enhance the stability of the generator frequency, operation closer to the serial resonance frequency is required, and quartz resonators with a small $\delta_{0}$ values hould be selected.If $\delta_{0}$ is large, then one should compensate static capacitance using an inductance connected in parallel, which is possible only in some serial resonance generators.

At frequencies from $800 \mathrm{kHz}$ to $30 \mathrm{MHz}$ or higher, if a generator has to be adjusted, a generator with an in-line resonator must be used. This scheme demonstrates stability at frequencies of up to $90 \mathrm{MHz}$. In the range from $800 \mathrm{kHz}$ to $90 \mathrm{MHz}$, one should use a generator with a resonator in a feedback loop if high power is required. Here, one should compensate static capacitance $C_{0}$ at frequencies above $30 \mathrm{MHz}$ by an inductance connected in parallel to the quartz.

According to equation (1), the reactive conductivity is capacitive in most of the spectrum and the resonator manifests inductive behavior only in the narrow frequency range from $f_{r}$ to $f_{a}$. At frequencies $f_{r}$ and $f_{a}$, the reactive conductivity component is zero. The lowest of these frequencies, $f_{r}$, is the resonance frequency, while the higher one, $f_{a}$, is the antiresonance frequency.

As to the other frequencies, $f_{s}$ and $f_{p}$, a conductivity maximum is observed at $f_{s}$ in the $L_{1}, C_{1}, R_{1}$ dynamic branch (Figure 1), while its minimum is observed at $f_{p} . f_{s}$ is referred to as the serial resonance frequency. It coincides with the mechanical resonance frequency of a piezoelectric resonator and thus characterizes most accurately its mechanical properties [1]:

$$
f_{s}=\frac{1}{2 \pi} \cdot \sqrt{L_{1} C_{1}}
$$

The $f_{p}$ value is referred to as the parallel resonance frequency:

$$
f_{p}=\frac{1}{2 \pi} \cdot \sqrt{\frac{1}{L_{1} C_{1}}\left(1+\frac{C_{1}}{C_{0}}\right)}
$$

As one can see from comparison of relationships (10) and (11), the inter-resonance difference $\Delta f=f_{p}-f_{s}$ is determined by the ratio of the capacitances [1]:

$$
m=C_{1} / C_{0}
$$

Usually, $m<<1$, so:

$$
\frac{f_{p}-f_{s}}{f_{s}}=\frac{1}{2} m
$$

In general, serial resonance frequency $f_{s}$ differs from resonance frequency $f_{r}$ and parallel resonance frequency $f_{p}$ differs from antiresonance frequency $f_{a}$; for $\mathrm{m} \ll 1, f_{s}=f_{r}$ 
and $f_{p}=f_{a}$ according to [1]. An estimate shows that the relative differences are negligibly low for piezoelectric quartz resonators $\left(\mathrm{m} \approx 4 \cdot 10^{-3}\right)$ :

$$
\frac{f_{p}-f_{s}}{f_{s}} \approx \frac{f_{p}-f_{a}}{f_{a}} \approx 2 \cdot 10^{-7}
$$

The frequencies $f_{m}$ and $f_{n}$, which correspond to the total conductivity maximum and minimum, also differ insignificantly from $f_{s}$ and $f_{r}$. Therefore, only two characteristic frequencies are mentioned in the literature instead of three pairs of characteristic frequencies [1,42]: serial or mechanical resonance frequency $f_{s}$ and parallel resonance frequency $f_{r}$.

Alongside resonance frequencies, another practically important characteristic of a piezoelectric resonator is its mechanical Q-factor determined according to (15):

$$
Q=\frac{2 \pi f_{s} L_{1}}{R_{1}}=\frac{1}{2 \pi f_{s} C_{1} R_{1}}=\frac{\pi}{\delta}
$$

where $\delta$ is the logarithmic decrement of decay.

The mechanical Q-factor characterizes the ability of resonators to store the accumulated mechanical energy [1, 42].

\section{Quartz crystal resonator}

Piezoelectricity can be defined [43] as electric polarization provided by mechanical stress in some crystals. It is proportional to the voltage and changes sign alongside with it. This situation determines the direct piezoelectric effect. It is closely related to the inverse effect of appearance of mechanical voltage in a piezoelectric crystal under electric polarization and the mechanical voltage value is directly proportional to the polarizing field. Both effects correspond to reversible physical phenomena. The "direct" term is applied to the first term only on historical grounds.

In the first half of the XX century, the research centers of the world studied the properties and possibility of practical application of piezoelectric crystals [43], which resulted in development of many useful devices. Besides, studying the properties of plates of Seignette salt crystals in order to use them for underwater signaling systems allowed detecting in 1918 some particular features of their electric behavior in the frequency range close to the mechanical resonance [43].A piezoelectric resonator was developed on the basis of these experiments and was used as a stabilizer, generator, and electric wave filter. The functioning of such devices includes a combination of direct and inverse effects. It was found in the above studies that quartz is the most suitable material for a resonator.

The first attempt to patent a piezoquartz resonator was made in 1918 [44], 38 years after the discovery of the piezoelectricity effect in 1880 by Pierre and Paul-Jacques Curie. In 1928, the first quartz clock was designed [3] that was used as a timing reference (time standard) [2] until atomic clocks appeared in the 1960s. 
Studies of piezoresonators provided scientific information on the nature of vibration in crystalline media and on the dynamic values of elastic and piezoelectric constants. Compound resonators were also designed, in which, e.g., a metallic rod was maintained in the resonance vibration state using a glued-on quartz piece. This method was used to determine elasticity constants and internal friction coefficients of various solid substances. Among the technical applications of resonating crystals, one can mention their practically universal use in broadcast transmitting stations both for direct frequency adjustment in the form of piezogenerators and indirectly, as monitors (control units) [1, 42].The combination in quartz of remarkably weak decay with piezoelectric properties pronounced well enough to respond to the frequency of electron tube generators and control them led to a technique for obtaining much more constant frequencies than in the case of just electric tuning. Cutting oriented quartz plates allows largely eliminating some distorting effects caused by the relationship between different vibrations and also the effect of temperature variation on frequency. This accuracy reaches its climax in a quartz clock where a vibrating quartz plate or ring replaces a pendulum and thus provides a time meter that is more accurate than the best astronomical clock [3, 43].

The main element of a piezoelectric resonator is a quartz vibrator. A quartz vibrator is usually a crystal element of a resonator equipped with electrodes and, if required, with wire current taps soldered onto it and used for its assembly in a holder [42, 45].

The features of vibrator design are primarily determined by the type of vibrations of the quartz element. The most characteristic features are the configuration of the electrode applied on the element, the choice of points of current tap fixation and their connection to the holder current leads. The best combination of electric and mechanical characteristics of resonators can be provided only in the case when the crystal is fixed in junction points, i.e., in its surface points that are not subject to displacement in the course of vibrations. As the crystal holder elements are also usually conductors that connect it to the external electric circuit, the metal electrode layer shape is determined by the necessity of applying the corresponding external voltage phase directly through the junction points. In most cases, every electrode, beside the main part that excites the given vibration type, is also equipped with branches, necks, or pockets connecting the main part with the current tap soldering points [2].

A characteristic example is a keyhole-shaped electrode (Figure 3) applied on piezoelements with through-thickness vibrations. The working electrode part is a disc in the crystal center, while a neck serves to supply electric voltage from fixation points usually located on the plate edge in the exit points of axis $Z^{\prime}$ and the quartz is fixed between two O-shaped rings $[2,5,45]$.

As pointed out above, quartz is the most suitable material for a piezoelectric vibrator. 


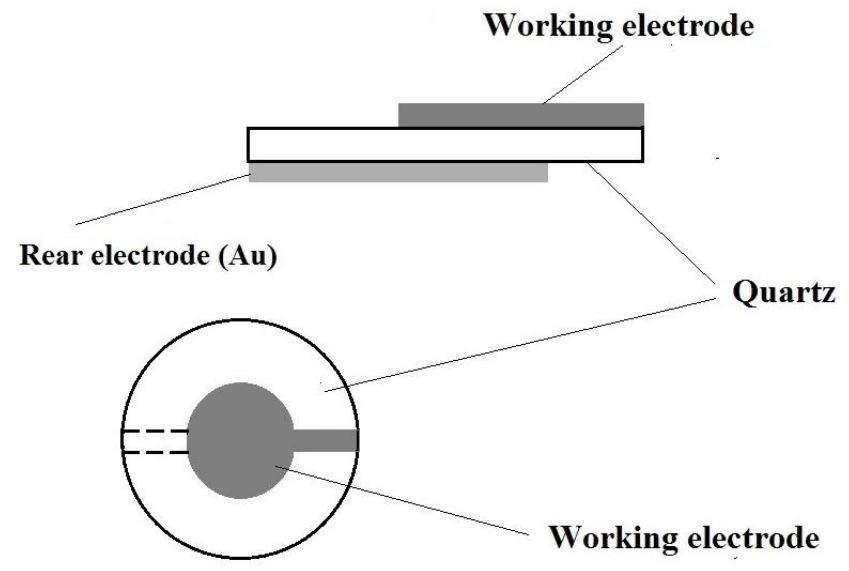

A

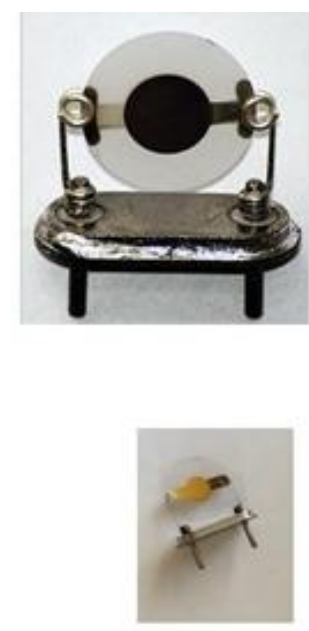

B

Figure 3. Quartz resonator. A. Scheme of a resonator with a "keyhole" - shaped electrode. B. Resonator outward appearance.

\section{Electrochemical quartz crystal microbalance (EQCM)}

At the turn of the $\mathrm{XX}-\mathrm{XXI}$ centuries, a technique was developed that allows one to use a quarts resonator in liquid media and combine quartz crystal microbalance and electrochemical research methods, i.e., electrochemical quartz crystal microbalance (EQCM) $[5,46]$. The first works employing the EQCM method appeared in the eighties of the twentieth century $[47,48]$.

Figure $4 \mathrm{a}$ shows a flow chart of two different methods of designing the EQCM measurement scheme. Initially, the frequency was determined by measuring the difference between a working quartz resonator in the measurement solution and a reference quartz, converting this difference to voltage, and recording the result on a computer. The suggested experimental setup requires no separate frequency meter and provides the time resolution sufficient for the majority of QCM applications. Intense development of electronics has enabled direct frequency measurements, which requires the QCM setup time resolution synchronized with the internal timing frequency: the higher the timing frequency, the better the time resolution. At present, frequency sensors with internal timing frequencies above $1 \mathrm{GHz}$ that are sufficient for obtaining millisecond resolution (for the quartz crystal frequency of $10 \mathrm{MHz}$ ) are standard.

The process of frequency measurement is presented in more detail in Figure $4 \mathrm{~b}$. A high frequency is applied to a quartz via a coaxial cable, where the working electrode side is connected to the screening coaxial cable armor. Simultaneously, the electrode output of a potentiostatis connected to the working electrode. In such a configuration, the working electrode is safely earthed. 


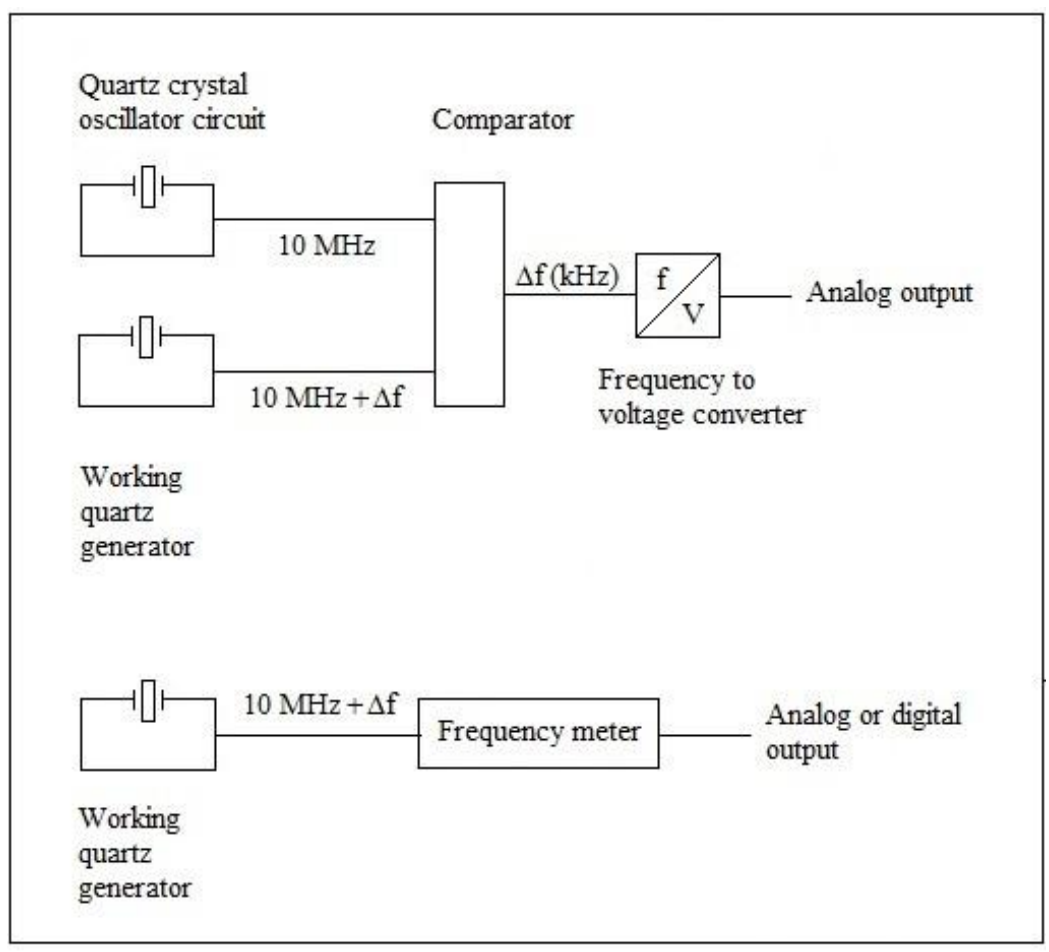

Figure 4a. Flow chart illustrating two frequency measurement methods.

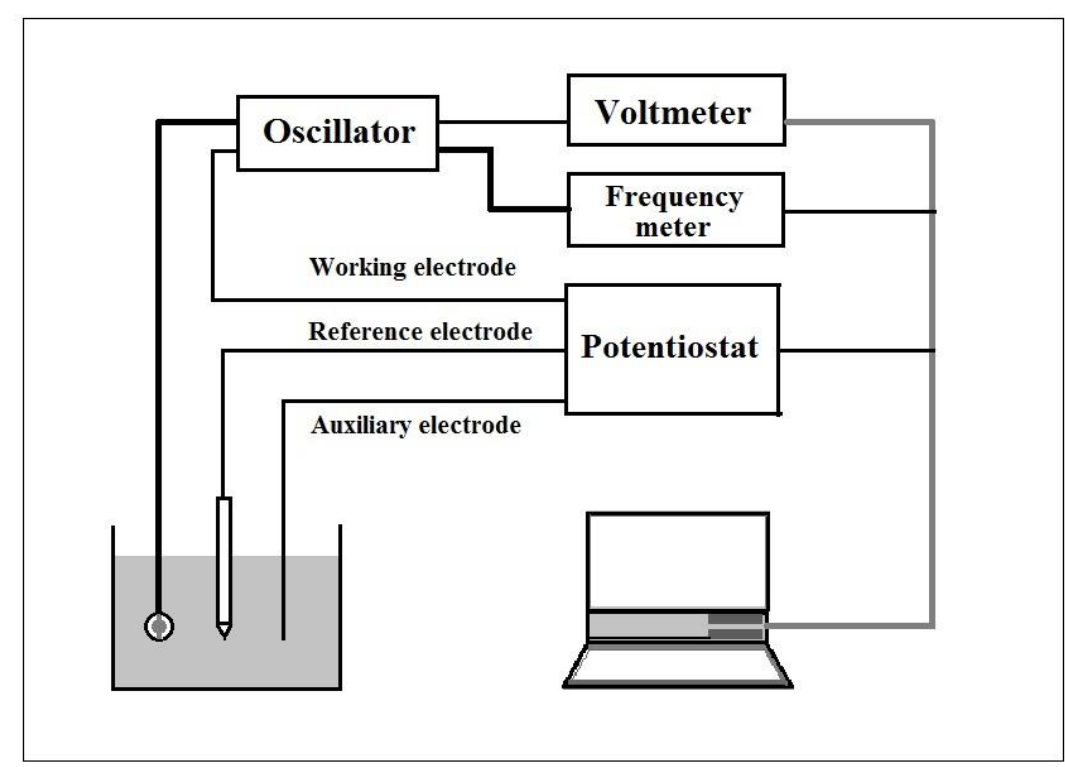

Figure 4b. Schematic diagram of a computer-controlled experimental EQCM setup.

The high frequency is measured using a central coaxial cable lead connected to the rear resonator electrode. In this setup, a commercial generator can be used that can operate a quartz crystal in the frequency range of 5 to $10 \mathrm{MHz}$ with parasitic capacitance compensation, which allows obtaining a high-frequency signal and measuring the voltage value that can be used to calculate the crystal resistance. The potentiostat is controlled using a computer that records the measured data. To increase the rate of data collection, 
one can temporarily store frequency data in the internal memory of a built-in frequency meter in the device and/or in a computer.

EQCM is calibrated by providing conditions for an increase and decrease of the mass on the working electrode, e.g., when copper is deposited from an acidic copper sulfate solution under the conditions of the $100 \%$ current efficiency $[5,49]$ with the mass variation calculated according to Faraday's law and with the resonator frequency shift measurement.

\section{Application of the quartz crystal microbalance method for metal surface studies}

The high sensitivity and effective absence of inertia of the QCM method favor its wide application, primarily, for studies of regularities of the initial stages of oxide film growth on metals upon adsorption of oxygen, water vapors, and other corrosive gases $[21,26,32,33,37]$.

Accurate interpretation of processes resulting in mass variation at the micromolecular level is difficult. However, the scope and quality of the experimentally obtained information can be significantly enhanced by simultaneous employment of other physical methods or (for gas phase QCM) application of ultrahigh vacuum setups for the formation of pure films on the metals being studied [21].

The kinetics of low-temperature $(\sim 300 \mathrm{~K})$ oxidation is characterized by fast and slow stages of oxide film growth. The kinetic regularities of adsorption processes obtained in a number of works allowed calculating the variation of the sticking coefficient of oxygen on metals characterizing the course of the process of oxygen atom capture as the surface is covered with adsorbed gas molecules (atoms). It appeared that the sticking coefficient is much smaller than unity at room temperature at initial time instants. For example, it is $3 \cdot 10^{-2}$ for aluminum films, $10^{-1}$ for copper, and $2 \cdot 10^{-3}$ for zinc. With time, the sticking coefficient further decreases by several orders of magnitude. A change in the sticking coefficient as dependent on the surface coverage rate is most often described by a complex curve, sometimes containing a maximum [21].

The initial stages of low-temperature oxidation in pure oxygen atmosphere have been studied in most detail on thin films of iron, aluminum, zinc, titanium, and some other metals [21, 43]. The fast initial interaction of oxygen with thin (100 $\AA)$ aluminum films at room temperature later turns to a slower stage, as the oxygen surface coverage approaches unity. Here, the resistance of aluminum film changes dramatically and the electronic work function passes a minimum. The proposed mechanism of the initial oxidation stages includes dissociative adsorption followed by intercalation of oxygen atoms into the metal lattice (exchange with aluminum atoms) resulting in the formation of several oxide layers $[21,52]$. Such oxygen intercalation can be considered as an intermediate process between chemisorption and formation of a phase oxide.

In $[53,54]$, the adsorption of organosilicon monomers (organosilanes) on the surface of freshly deposited aluminum was studied, along with the effect of surface organosilicon (methyl-, amino-, vinyl-, and phenyl-containing) layers on the oxide film hydration. It was 
shown that if the surface was coated with an organosilicon layer, the surface oxide layer hydration rate decreased more than by half.

Zinc oxidation in oxygen at $p_{\mathrm{O}_{2}}=10^{-1}$ Torrat the room temperature is described by a two-stage logarithmic law [21,50]. The first stage, 20-30 s long, is related to surface "oversaturation" with chemisorbed ionized oxygen followed by its regrouping into a phase oxide layer. The slower second stage is determined by phenomena of diffusion into the growing oxide film. If the oxygen pressure is decreased to $c a \cdot 10^{-4}$ Torr, the oxidation rate of zinc linearly depends on the pressure.

A model was suggested on the basis of the functional dependence of the sticking coefficient on the amount of absorbed oxygen. According to this model, oxygen chemosorption results in formation of $\mathrm{ZnO}$ is let nuclei at a higher rate at the corners than on a pure metal surface. With time, the nuclei grow and form a stable film with the thickness of up to $30 \AA$ on the entire metal surface [51].

Studies of oxygen chemisorption on zinc show that electric field created by intercalated oxygen enhances the bonding energy of the incumbent cations with the surface, i.e., creates an additional potential barrier and hampers the removal of these cations into the reaction zone. Ionized oxygen chemisorbed on these cations forms a field of opposite polarity and promotes their transition into the oxidation zone. A development of these concepts has shown that there is a direct relationship between phenomena observed in the case of oxygen adsorption on a freshly formed metal surface in vacuum and appearance of a transition region between the potentials of passivation initiation and full passivation in aqueous electrolytes. Electrostatic fields formed in both cases when a submonolayer oxygen amount is adsorbed cause a decrease in the electronic work function and cutoff of the anodic dissolution current (ionic current from the metal or electronic current from the electrolyte) $[21,50]$.

At a low temperature $(77 \mathrm{~K})$, the growth of metal oxide on potassium films obtained in ultrahigh vacuum practically stops after formation of a few atomic layers. At room temperature, the kinetics of potassium oxidation by oxygen $\left(10^{-8}\right.$ to $10^{-9}$ Torr $)$ follows a linear law, i.e., oxygen atoms diffuse into the metal bulk at a rate comparable to the rate of their sorption on the potassium film surface [21].

If $\mathrm{CO}$ interacts with titanium films of various mass $(0-8 \AA)$, the $\mathrm{CO} / \mathrm{Ti}$ atomic ratio in the film is very close to the theoretical $\mathrm{CO} / \mathrm{Ti}$ ratio $=0.5$. As $\mathrm{CO}$ is dissociatively adsorbed on titanium, one can conclude that each titanium atom is bonded with $\mathrm{C}$ and $\mathrm{O}$ atoms. "Saturation" is achieved more slowly than with oxygen, apparently because C atoms remain on the surface and block the further adsorption of $\mathrm{CO}$ molecules [55].

A study of the sputtering process in case of argon etching of electrodeposited oxidized nickel and iron allowed determining some kinetic regularities of oxide degradation. In the case of $\mathrm{NiO}$, only a single degradation process was observed. Two different degradation processes were distinguished for oxidized iron [21]. 
Water molecules on corroding metals ( $\mathrm{Fe}, \mathrm{Zn}, \mathrm{Cu}, \mathrm{Al}$, etc.) are sorbed irreversibly. The metals react with water vapors more slowly than with oxygen. The observed oxidation is a multistage process including dissociation of water molecules, oxygen penetration into the surface metal layer, and water adsorption on the oxide formed. Studies of water adsorption on metal surface show that more than a single water monolayer is adsorbed on the metal surface even at a relative humidity of $50 \%$, while an increase in the relative humidity to $80 \%$ results in an increase in the thickness of the adsorbed water layer to more than 10 molecular layers $[21,27,50]$.

After the initial fast stage of water adsorption on iron and aluminum films evaporated in ultrahigh vacuum, the gas phase contains hydrogen (as a result of dissociation of sorbed water molecules and desorption of the major fraction of hydrogen into the gas phase, though its small fraction can be dissolved in the metal). At the initial stages, a mass equivalent to four $\mathrm{FeO}$ layers is registered at the initial stages. It grows in 30-60 min to ten equivalent layers of $\mathrm{FeO}$, after which the oxidation rate drops abruptly. The formation of an oxide film on iron is interpreted as a gradual release of hydrogen atoms in the gas phase by a water molecule sorbed on the iron surface. Afterwards, oxygen is intercalated into the crystal lattice; here, a pure iron surface is regenerated. On aluminum, water adsorption at room temperature leads to appearance and lateral growth of oxide layer nuclei, on which water is further adsorbed in the form of dipoles by a first order reaction [21, 26, 27, 50].

The interaction of zinc with water vapors may result in the formation of such surface compounds as $\mathrm{ZnO}$ at $P / P_{0}<0.3$ or $\mathrm{Zn}(\mathrm{OH})_{2}$ at $P / P_{0}>0.6$. A change in the electronic work function (EWF) in the case of water vapor adsorption on zinc (a decrease in EWF when less than a monolayer is adsorbed and a growth at high surface coverages) indicates that water oxygen is adsorbed at initial stages of interaction on silver, same as on zinc and iron, and is intercalated into the metal lattice. Zinc preconditioning in deoxygenated water vapors hinders the further metal oxidation by dry air or pure oxygen $[21,50]$.

Combined or alternating exposure of an iron layer sputtered in vacuum $\left(10^{-4}\right.$ to $10^{-5}$ Torr) to water vapors and oxygen leads to the formation of a passivating film that obviously represents a coordination-saturated hydrated surface oxide with $\mathrm{FeOOH}$ composition $[29,56]$.

A change in the mass and resistance in the case of adsorption interaction between water vapors and thin copper, aluminum, and nickel films (500-2000 $\AA$ ) oxidized in air allows one to conclude that an increase in the water vapor pressure (with an increase in the relative humidity) can cause a change in the localization of adsorbate-adsorbent bonds (the number of metal-water bonds decreases and the number of oxide-water bonds grows) $[21,50]$.

Thus, the QCM method gained wide recognition in experimental studies on phenomena at gas-solid, gas-liquid-solid, and liquid-solid interfaces [5, 6, 21].Owing to its very high sensitivity, simplicity, and possibility of experimenting with diverse materials and media, the method allows fast acquisition of high-quality scientific information on processes occurring on the surface and is very promising for metal corrosion studies. 


\section{Application of the QCM and EQCM methods in studies of corrosion processes}

The EQCM method has been widely used for in situ studies of various corrosion processes by measurement of mass variation due to metal dissolution or film growth on the surface $[5,6,40,57]$. Despite the obvious advantages of the QCM method, interpretation of EQCM data requires in a number of cases that additional research methods be used that allow analyzing the surface, e.g., XPS, IR spectroscopy, SEM, AFM, and electrochemical impedance spectroscopy [5]. Hereafter, the use of EQCM will be considered for studying the processes of atmospheric corrosion, corrosion in solutions, behavior of corrosion inhibitors, and passive film growth on metals.

\subsection{Atmospheric corrosion of metals}

Exposure of a metal to a wet atmosphere results in formation of corrosion products that usually remain on the surface. The corresponding increase in the sample mass can be monitored by QCM used by different researchers in investigations on atmospheric corrosion of metals $[5,6,21,27,50]$. Papers $[27,58]$ describe a setup consisting of a test chamber providing a continuous gas flow. Besides, this setup combines QCM for studying the kinetics of the occurring reactions and an IR spectrometer (Fourier Transform IR spectroscopy (FTIR)) for obtaining information about the occurring chemical reactions. $1 \mathrm{~nm}$ thick oxide films on copper were studied using FTIR and $0.2 \mathrm{~nm}$ thick oxide films were studied by QCM. The effect of relative humidity and content of sulfur dioxide, ozone, and nitrogen oxides in the atmosphere on copper corrosion was also studied. A built-in atomic force microscope (AFM) in the working setup allowed estimating the topographic changes due to atmospheric corrosion of copper [58]. It was found that the overall mass variation was greater when $\mathrm{SO}_{2}$ was added to air with a humidity of $80 \%$, but the largest effect was observed when ozone was added [5, 27, 58]. The amounts of sulfate, water, and copper oxide on the surface were estimated using FTIR to identify the corrosion products formed under various conditions. A similar approach was used to study the formation of ultrathin polysiloxane films on oxidized iron under glow discharge with subsequent estimation of the anticorrosion properties of the thin films obtained [5, 6].

The practical application of QCM for corrosion monitoring at an automatic telephone switching office was described in [59]. QCM sensors metallized with various metals, such as copper, silver, nickel, and gold, were placed in air ducts and the mass variation was measured for several months. At the end of a test, the sensor surface was studied using Auger electron spectroscopy. It was shown that corrosion rates were much higher when air was being removed from the system than when it was collected from the atmosphere for addition into the system $[5,59]$.

Thus, the QCM method is successfully used in studies of atmospheric corrosion and allows high precision determination in situ of a metal mass variation under atmospheric conditions. Application of the EQCM method can allow studying the metal corrosion in liquid media. 


\subsection{Metal corrosion in electrolytes}

EQCM allows measuring metal corrosion rates in electrolytes. In the course of formation of soluble corrosion products, linear dependence between the variation of the piezoresonance sensor frequency and mass variation due to corrosion was observed, which was confirmed for cobalt in an aerated sulfuric acid solution and for copper in hydrochloric acid $[5,6]$. The EQCM method allows determining the anodic current as a function of potential, as the partial anodic current density is proportional to the mass loss rate according to Faraday's law.

In the studies of uniform iron corrosion in deaerated neutral borate and phosphate electrolytes, the quartz electrode was placed onto the wall of a flow-through cell. A linear relationship between the frequency variation and time was observed under open-circuit conditions, which indicated that only soluble corrosion products formed. The corrosion rate was higher in phosphate solutions than in solutions containing borate ions. It was shown quantitatively that addition of chloride caused an increase in the corrosion rate [5].

EQCM was used in combination with other electrochemical methods, such as electrochemical impedance spectroscopy or cyclic voltammetry. Some researchers used EQCM in combination with detection of reaction products using an in situ technique similar to the rotating ring disc electrode [5, 62]. A quartz electrode was incorporated into the electrochemical cell wall and was surrounded by a ring or split ring electrode separated from the disc by a narrow gap. The electrolyte flow directed normally to the disc provided reproducible mass transport conditions. EQCM was used to study copper dissolution in chloride media by simultaneous measurement of sample mass variation and the amount of dissolved monovalent reaction products. EQCM was used in combination with electrochemical impedance spectroscopy in studies of copper dissolution in $\mathrm{NaCl}$ and $\mathrm{NaHCO}_{3}$ solutions using a scheme that allowed identifying intermediate products of surface reactions. Studies of anodic dissolution of an $\mathrm{Au}-\mathrm{Cu}$ alloy in a $\mathrm{Na}_{2} \mathrm{SO}_{4}$ solution with simultaneous EQCM and electrochemical impedance spectroscopy measurements showed that the frequency shift due to selective $\mathrm{Cu}$ dissolution was smaller than the value calculated by Faraday's law. This effect was explained by the fact that water penetrating the porous surface structure is removed from the surface during the dissolution. Studies of the corrosion rate of $\mathrm{Au}-\mathrm{Pd}$-In alloys in physiological solutions showed an increase in the alloy mass under open circuit conditions related to accumulation of corrosion products. An increase in the mass was also observed on sputtered $\mathrm{Fe}-\mathrm{Cr}-\mathrm{Ni}-\mathrm{Ta}$ alloys under exposure to a neutral chloride-containing solution [5].

In $[53,54,65,67]$, EQCM was used to study the effect of surface self-organizing nanolayers on local metal dissolution and showed that the EQCM method could yield information in studies of not only uniform, but also local corrosion and that siloxane nanolayers could successfully inhibit metal dissolution.

Thus, electrochemical quartz crystal microbalance allows studying the mechanisms of both uniform and local metal corrosion. 


\subsection{Studies of metal corrosion inhibitors}

It is known that the EQCM method is quite suitable for studying the behavior of corrosion inhibitors [5] and for estimating their corrosion protection efficiency. Thus, studies of the corrosion behavior of nickel in $\mathrm{HClO}_{4}$ solutions in the presence of various corrosion inhibitors [68] showed that the measured value equaled the difference between the mass of the film formed and the dissolved metal mass. The authors described predominant nickel dissolution that was confirmed by a decrease in the mass. Tributylbenzylammonium iodide was the most efficient of the inhibitors studied, while acridine promoted dissolution [68]. EQCM was also used for studying the inhibition of copper corrosion [33, 40, 65].

Studies of biofilm formation on iron showed that bacterial adhesion resulted in a measurable mass increase that depended on the presence or absence of biocides. Hydrophilic and ionic biocides proved to be more efficient in decreasing the adhesion of bacteria than hydrophobic biocides [5].

In the presence of corrosion inhibitors forming surface films, reliable interpretation of data on mass variation requires the use of additional methods, such as cyclic voltammetry [49] or electrochemical impedance spectroscopy [5]. For example, studying the film formation on copper in the presence of benzotriazole demonstrated good agreement between the data obtained by the electrochemical impedance spectroscopy and EQCM techniques. Studying the effect of a phosphonate corrosion inhibitor and flow rate in the case of sediment formation on a gold piezoquartz electrode placed into the wall of a flowthrough channel and exposed to mineralized water showed that scale formation was promoted by electrochemical polarization and addition of an inhibitor or an increase in the flow rate resulted in a decrease in the positive mass variation due to scale formation [5].

Owing to high QCM sensitivity in mass measurements, this method is used especially often in studies of corrosion inhibitor adsorption on metals [28, 30, 36, 40, 45, 46, 60, 61]. For example, the adsorption behavior of organic inhibitors on iron and gold was studied in neutral perchlorate and borate solutions [45, 46]. After an inhibitor was added to a perchlorate solution, the mass was also measured using rotating EQCM to provide reproducible mixing and using EQCM in combination with ellipsometry. Addition of an inhibitor to the solution resulted in a positive frequency shift indicating a decrease in the electrode mass. The results were interpreted assuming that the adsorbed inhibitor displaces water from the region near the electrode surface. Indeed, the QCM response in these electrolytes includes a solution region with a thickness of about $17 \mathrm{~nm}[5,6]$. The adsorption isotherms of sodium phenylanthranilate from a borate buffer solution on a gold electrode surface obtained by EQCM and ellipsometry methods are similar in shape and are described by the Frumkin equation [28].

Thus, analysis of the literature shows that the QCM method is successfully used in studies of the mechanism of action of corrosion inhibitors and mechanisms of processes at the metal interface in the presence of inhibitors. 
6.4. Application of EQCM in studies of processes of growth and dissolution of oxide films on metals

Passive films on metals and alloys grow due to the interaction of oxygen anions in the solution with the surface (Figure 5) [5]. This results in an increase in the electrode mass, which can be monitored using EQCM. For a known film stoichiometry, comparison of the measured mass variation with transferred charge allows separating the reactions of film formation and metal dissolution [5]. Assuming that an anodic potential is applied to metal $\mathrm{M}$ in its passive state with the potential difference resulting in formation of oxide $\mathrm{MO}_{v}$ (where $v$ is the stoichiometric coefficient), the mass of metal oxidized at the metal/oxide interface, $\Delta m_{I}\left(\mathrm{~g} / \mathrm{cm}^{2}\right)$, proves to be proportional to the charge density $q\left(\mathrm{C} / \mathrm{cm}^{2}\right)$ determined as (16):

$$
\Delta m_{I}=\frac{q M_{m}}{n F}
$$

where subscript $I$ indicates the metal/oxide interface, $M_{m}$ is the atomic mass of the metal, $n=2$ is the number of electrons participating in the reaction, and $F$ is Faraday's constant.

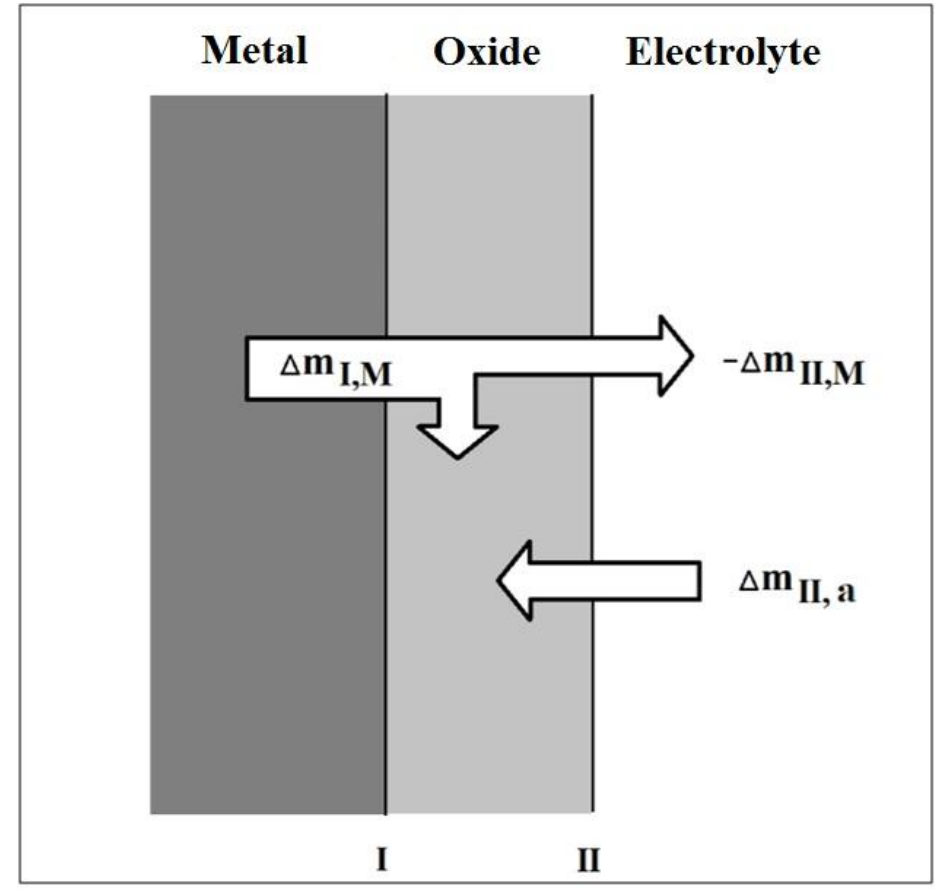

Figure 5. Schematic diagram of partial reactions related to the growth of anodic metal oxide and passive film dissolution.

Only the $R_{g}$ fraction of the oxidized metal remains in the film, while the rest of it is dissolved at the film/solution (II) interface (Figure 5). The mass of fraction $R_{g}$ is determined by expression (17): 


$$
R_{g}=\frac{\Delta m_{I, M}+\Delta m_{I I, M}}{\Delta m_{I, M}}=1+\frac{\Delta m_{I I, M}}{\Delta m_{I, M}}
$$

In equation (18), $\Delta m_{I I, M}$ is negative, as all the values resulting in the film growth are assumed to be positive. This definition corresponds to EQCM measurement, namely, ingression (supply) of the substance into the film, results in an increase in the mass, and is assumed to be positive, while the film dissolution corresponds to negative values [5]. The measured mass variation $\Delta m$ includes a mass increase due to incorporation of anions into the growing film $\Delta m_{I I, A}$, while $\Delta m_{I I, M}$ is the metal ion loss due to dissolution at the film/solution interface, equation (18):

$$
\Delta m=\Delta m_{I I, \text { anion }}+\Delta m_{I I, M}
$$

Knowing the film stoichiometry, one can derive the relationship between the values of $\Delta m_{I I, A}, \Delta m_{I, M}$, and $\Delta m_{I I, M}$ :

$$
\frac{M_{M}}{v} \Delta m_{I I, \text { anion }}=M_{0}\left(\Delta m_{I, M}+\Delta m_{I I, M}\right)
$$

These relationships can be used to determine the fraction of the film growth according to $(20)[5]$ :

$$
R_{g}=\frac{M_{M}+n F(\Delta m / q)}{M_{M}+v M_{0}}
$$

Thus, the measured charge-mass ratio allows one to directly estimate the fraction that determines the oxide film growth (21) [5]. Similarly, one can determine the differential or instantaneous growth fraction $r_{g}$ (equation (21) that can be used for online characterization of film growth, e.g., in metal anodic treatment [5].

$$
r_{g}=\frac{\left.M_{M}+n F[\partial m / \partial t) / i\right]}{M_{M}+v M_{0}}
$$

Variation of oxide film mass $\Delta m_{I I}$ can be determined from the measured mass and charge variation (equation (22):

$$
\Delta m_{\text {film }}=\Delta m_{I I, \text { anion }}+\Delta m_{I, M}+\Delta m_{I I, M}=\Delta m+\Delta m_{I, M}=\Delta m+q \frac{M_{M}}{n F}
$$

In [37], EQCM was used to study the initial stages of iron passivation in neutral solutions and it was shown that joint application of the in situ quartz resonator method and pulsed chronoamperometry allowed determining the kinetics of the initial period of passive film growth on iron. The film thickness measured with account for the "true" electrode surface area (about $1 \mathrm{~nm}$ ) reasonably coincided with the thickness of the oxide layer formed on metallurgic metal. It was found that a primary passive film formed at iron prepassivation potentials in a borate buffer solution $(\mathrm{pH} 6.7)$; the average film thickness reached several monolayers of oxide/hydroxide compounds at less negative $(-0.3 \mathrm{~V})$ 
potentials and did not exceed a single monolayer at more negative $(-0.4 \mathrm{~V})$ potentials. In the initial period, the dependences of the passive layer thickness on time were linear in logarithmic coordinates, with the slope independent of the potential. It was found that the primary film growth at the potentials of iron passivation and prepassivation was described by the same kinetic equation. The start of formation of a continuous barrier layer on iron corresponded to a minimum in the dependence of the fraction of the anodic oxide formation reaction on time $\left(g_{i}\right)$. According to this criterion, the time of the smallestthickness barrier layer formation is $2-3 \mathrm{~s}$ at the passivation potential $(E=0 \mathrm{~V})$, while no compact layer of oxide/hydroxide compounds of $\mathrm{Fe}^{2+}$ was formed at the potentials of metal prepassivation. This was confirmed by the shape of the dependences of $g_{i}$ on time [37]. Besides, it was shown that addition into borate buffer solution $(\mathrm{pH}$ 6.7) of a metal hydrogenation promoter (thiocyanate ions) prevented the formation of a primary passive film and accelerated iron dissolution at the prepassivation potential. Here, a relatively thick (at least $2 \mathrm{~nm}$ ) layer formed on the electrode surface has no inhibiting effect on the metal dissolution [37].

\section{Conclusion}

The QCM and EQCM techniques find wide application in studies of processes at the gas-solid, gas-liquid-solid, and liquid-solid interfaces and are versatile in situ methods for the estimation of mass variation in time. They reflect the processes of adsorption, metal dissolution, and growth of protective films or corrosion products. Owing to their very high sensitivity, simplicity, and possibility of research in various media on many materials, this method allows the fast acquisition of qualitatively new scientific information. It is of particular interest to solve scientific problems by combined employment of QCM with other methods of physicochemical surface analysis. Such methods include XPS, scanning electron microscopy, atomic force microscopy, Auger and IR spectroscopy, and also electrochemical impedance spectroscopy, voltammetry, and chronoamperometry. For example, a combination with XPS allows studying the kinetics of growth of oxide films of multicomponent alloy elements with very different chemical properties.

The efficiency of the QCM method for studying oxidation, corrosion, interaction of inhibitors with metals, protective and destructive characteristics of thin-film coatings is obvious. In fact, QCM is successfully used in corrosion to solve a variety of problems. Undoubtedly, the further application of the QCM method for studying corrosion and metal protection will allow considerably expanding the understanding of the mechanisms of corrosion processes. 


\section{References}

1. V.V. Malov, Piezorezonansnye datchiki (Piezoresonance sensors), Moscow: Energoatomizdat, 2nd ed., 1989, 272 pp. (in Russian).

2. D. Johannsmann, The Quartz Crystal Microbalance in Soft Matter Research. Fundamentals and Modeling, New York, Dordrecht, London: Springer Cham Heidelberg, 2015, 287. doi: 10.1007/978-3-319-07836-6

3. W.A. Marrison, The Crystal Clock, Proc. Natl. Acad. Sci. U. S. A., 1930, 16, 496-507. doi: $10.1073 /$ pnas.16.7.496

4. G. Sauerbrey, The use of quarts oscillators for weighing thin layers and for microweighing, Z. Angew. Phys., 1959, 155, 206-222. doi: 10.1007/BF01337937

5. C.-O.A. Olsson and D. Landolt, Electrochemical Quartz Crystal Microbalance, in Analytical Methods in Corrosion Science and Engineering, Ed.: P. Marcus, F. Mansfeld, FL, USA: CRC Press, Taylor \& Francis Group, LLC, Boca Raton, 2006, 733-751. doi: 10.1201/9781420028331

6. D. Landolt and S. Mischler, Tribocorrosion of passive metals and coatings, Woodhead Publishing Limited, Oxford - Cambridg - Philadelphia, New Delhi, 2011, 554 pp.

7. G.E. McGuire, Auger electron spectroscopy reference manual. A Book of Standard Spectra for Identification and Interpretation of Auger Electron Spectroscopy Data, Springer Science + Business Media, New York, 1979, 143 pp. doi: 10.1007/978-14757-1702-0

8. L.P. Podgornova, V.F. Shirokov, L.P. Kazanskii and Yu.I. Kuznetsov, X-ray photoelectron spectroscopy of corrosion inhibitors. 4. X-ray photoelectron investigation of the absorption of benzimidazole on copper surfaces in phosphate solutions, Bull. Acad. Sci. USSR, Div. Chem. Sci. (Engl. Transl.), 1987, 36, no. 10, 2057-2060. doi: 10.1007/BF00961985

9. L.P. Kazanskii, Electron spectroscopy of corrosion inhibitors on metal surface. Part 1, Korroz.: mater., zashch. (Corrosion: materials, protection), 2007, no. 1, 40-48 (in Russian).

10. N.P. Andreeva, A.V. Larionov, O.Yu. Grafov, O.A. Golubchikov, L.P. Kazanskii and Yu.I. Kuznetsov, The adsorption of 5,10,15,20-tetrakis $(N$-methylpyridyl$\left.4^{\prime}\right)$ porphinetetratosylate on the surface of nickel from an aqueous solution, Prot. Met. Phys. Chem. Surf., 2018, 54, no. 7, 1276-1283. doi: 10.1134/S2070205118070031

11. N.P. Andreeva, L.P. Kazanskii, I.A. Selyaninov, Yu.I. Kuznetsov and V.A. Ostrovskii, Adsorption of 5-phenyltetrazole on iron and its inhibition of the dissolution of lowcarbon steel in a neutral solution, Prot. Met. Phys. Chem. Surf., 2009, 45, no. 7, 806-811. doi: 10.1134/S2070205109070107

12. R.F. Egerton, Physical Principles of Electron Microscopy. An introduction to TEM, SEM, and AEM, Springer Science + Business Media Inc., N.Y., 2005, 202 pp. doi: 10.1007/978-3-319-39877-8 
13. I.M. Romanenko, A.A. Viryus, V.A. Churin, A.S. Deyanov and A.S. Ivanov, Estimation of detection limits in electron probe X-ray microanalysis, J. Surf. Invest.: X-Ray, Synchrotron Neutron Tech., 2012, 6, 616-622. doi: $\underline{10.1134 / \mathrm{S} 1027451012070105}$

14. A.V. Maksimova, A.V. Kozinkin and V.G. Vlasenko, Electron and atomic structure of a polymer cluster material based on the $\mathrm{Mn}_{12} \mathrm{O}_{12}(\mathrm{OAc})_{16}\left(\mathrm{H}_{2} \mathrm{O}\right)_{4}$ molecular magnetic material, Fundamental'nye issledovaniya (Fundamental Research), 2016, 8, 50-54 (in Russian).

15. Fourier transform infrared spectroscopy. Applications to chemical systems, 4, Eds.: J.R. Ferraro and L.J. Basile, Academic Press, Orlando - San Diego - New York London - Toronto - Montreal - Sydney - Tokyo, 1985, 406 pp.

16. Fourier transform infrared characterization of polymers, Ed.: H. Ishida, Plenum Press, New York - London, 1984, 449. doi: 10.1007/978-1-4684-7776-4

17. D.O. Flamini, M. Trueba and S.P. Trasatti, Aniline-based silane as a primer for corrosion inhibition of aluminium, Prog. Org. Coat., 2012, 74, 302-310. doi: 10.1016/j.porgcoat.2011.11.011

18. M. Gettings and A.J. Kinloch, Surface characterization and adhesive bonding of stainless steels. I - The surface characterization of the steels, Surf. Interface Anal., 1979, 1, no. 5, 165-172. doi: 10.1002/sia.740010506

19. A.J. Kinloch, Adhesion and adhesives. Science and technology, Springer-science + business media, B.V., 1987, 441 pp. doi: 10.1007/978-94-015-7764-9

20. G.V. Vol'f, Yu.P. Chuburin, D.V. Fedorov and V.N. Strocov, An asymptotic approximation of multiple-scattering theory in very-low-energy electron diffraction from a metal surface, Phys. Solid State, 1999, 41, no. 12. 2105-2108. doi: $10.1134 / 1.1131129$

21. P.V. Strekalov, A.A. Mikhailov and M.N. Zasul'skaya, Method of quartz crystal microbalance and study of corrosion processes on metals, Zashchita metallov (Protection of Metals), 1983, 19, no. 2, 179-195 (in Russian).

22. Vacuum Microbalance Techniques, Ed.: R.F. Walker, Plenum Press Media, New York - London, 1962, 2, 179. doi: 10.1007/978-1-4899-6285-0

23. Vacuum Microbalance Techniques, Eds.: C.H. Massen and H.J. van Beckum, Plenum Press Media, New York - London, 1970, 7, 238. doi: 10.1007/978-1-4757-0725-0

24. Vacuum Microbalance Techniques, Ed.: A.W. Czanderna, Plenum Press Media, New York - London, 1971, 8, 251. doi: 10.1007/978-1-4757-0133-3

25. E. Robens, Sh. Amarasiri, A. Jayaweera and S. Kiefer, Balances. Instruments, Manufacturers, History, Springer, New York - Dordrecht - London - Heidelberg, 2014, 30. doi: $10.1007 / 978-3-642-36447-1$

26. D.A. Buttry and M.D. Ward, Measurement of interfacial processes at electrode surfaces with the electrochemical quartz crystal microbalance, Chem. Rev., 1992, 92, no. 6, 1355-1379. doi: $\underline{10.1021 / \mathrm{cr} 00014 \mathrm{a} 006}$ 
27. C. Leygraf, I.O. Wallinder, J. Tidblad and T. Graedel, Atmospheric Corrosion, 2nd Ed., John Wiley \& Sons Inc., Hoboken, New Jersey, 2016, 397.

28. Yu.I. Kuznetsov, L.B. Maksaeva, M.A. Petrunin and N.P. Andreeva, Adsorption of sodium phenylanthranylate on gold electrode from aqueous solution, Russ. J. Electrochem., 2009, 45, no. 11, 1340-1344. doi: 10.1134/S1023193509110044

29. M.A. Petrunin, L.B. Maksaeva, T.A. Yurasova, E.V. Terekhova, M.A. Maleeva, V.A. Kotenev, E.N. Kablov and A.Yu. Tsivadze, Formation of organosilicon selforganizing nanolayers on an iron surface from vapor phase and their effect on corrosion behavior of metal, Prot. Met. Phys. Chem. Surf., 2015, 51, no. 6, 1010-1017. doi: $10.1134 / \mathrm{S} 2070205115060179$

30. N.N. Andreev, O.A. Goncharova, N.P. Andreeva, L.B. Maksaeva, M.A. Petrunin and Yu.I. Kuznetsov, Adsorption of vapors of IFKhAN-118 volatile inhibitor on iron and steel, Korroz.: mater., zashch. (Corrosion: materials, protection), 2016, no. 2, 28-31. (in Russian).

31. T. Nomura and T. Nagamune, Internal electrolytic determination of silver in solution with a piezoelectric quartz crystal, Anal. Chim. Acta, 1983, 155, 231-234. doi: 10.1016/S0003-2670(00)85597-X

32. S. Bruckenstein and M. Shay, Experimental aspects of use of the quartz crystal microbalance in solution, Electrochim. Acta, 1985, 30, 1295-1300. doi: $10.1016 / 0013-$ $\underline{4686(85) 85005-2}$

33. S.N. Ovchinnikova, T.P. Aleksandrova and A.A. Weiss, Copper corrosion in acid chloride solutions: a quartz microgravimetry study, Russ. J. Electrochem., 2005, 41, 1185-1190. doi: 10.1007/s11175-005-0200-2

34. D. Landolt, Corrosion and surface chemistry of metals, EPFL Press Lausanne, Switzerland, 2007, 614 pp.

35. M.A. Petrunin, L.B. Maksaeva, T.A. Yurasova, E.V. Terekhova, M.A. Maleeva, A.A. Shcherbina, V.A. Kotenev, E.N. Kablov and A.Yu. Tsivadze, The effect of selforganizing vinyl siloxane nanolayers on the corrosion behavior of aluminum in neutral chloride-containing solutions, Prot. Met. Phys. Chem. Surf., 2014, 50, no. 6, 657-664. doi: $10.1134 /$ S2070205114060161

36. G. Vastag, A. Shaban, M. Vranes, A. Tot, S. Belic and S. Gadzuric, Influence of the $\mathrm{N}-3$ alkyl chain length on improving inhibition properties of imidazolium-based ionic liquids on copper corrosion, J. Mol. Liq., 2018, 264, 526-533. doi: 10.1016/j.molliq.2018.05.086

37. A.I. Marshakov, A.A. Rybkina, L.B. Maksaeva, M.A. Petrunin and A.P. Nazarov, A study of the initial stages of iron passivation in neutral solutions using the quartz crystal resonator technique, Prot. Met. Phys. Chem. Surf., 2016, 52, 936-946. doi: 10.1134/S2070205116030205

38. A.W. Czanderna and R. Vasofsky, Surface studies with the vacuum microbalance, Progr. Surf. Sci., 1979, 9, no. 2, 45. doi: 10.1016/0079-6816(79)90007-8 
39. C. Lu and A.W. Czanderna, Application of piezoelectric quartz crystal microbalance, ELSEVIER, Amsterdam - Oxford - New York - Tokyo, 1984, p. 393.

40. S. Mischler, S. Barril and D. Landolt, Fretting corrosion behaviour of Ti-6Al4V/PMMA contact in simulated body fluid, Tribol.-Mater., Surf. Interfaces, 2009, 3, no. 1, 16-23. doi: $10.1179 / 175158309 \times 408333$

41. A.W. Warner and C.D. Stockbridge, Mass and thermal measurement with resonating crystalline quartz in vacuum microbalance techniques, Eds.: R.F. Walker and H.S. Peiser, Springer Science + Business Media, New York, 1962, 2, 71-93. doi: 10.1007/978-1-4899-6285-0_7

42. M.I. Yaroslavskii and A.G. Smagin, Konstruirovanie, izgotovlenie $i$ primenenie kvartsevykh rezonatorov (Design, Manufacturing, and Application of Quartz Resonators), Energiya, Moscow, 1971, 168 pp. (in Russian).

43. W.G. Cady, Piezoelectricity: an introduction to the theory and applications of electromechanical phenomena in crystals, McGraw - Hill, New York - London, 1946, $806 \mathrm{pp}$.

44. A.M. Nicholson, Generating and transmitting electric currents, U.S. Patent 2.212.845, Granted Aug 27, 1940, Filed: Apr 10, 1918.

45. A. Arnau, Piezoelectric Transducers and Applications, Springer - Verlag, Berlin Heidelberg, 2004, p. 12. doi: 10.1007/978-3-662-05361-4_6

46. G. Mishra, A. Sharma and S. Bhand, Ultrasensitive detection of streptomycin using flow injection analysis - electrochemical quartz crystal nanobalance (FIA-EQCN) biosensor, Biosens. Bioelectron., 2015, 67, 532-539. doi: 10.1016/j.bios.2014.09.033

47. T. Nomura, Single-drop method for determination of cyanide in solution with a piezoelectric quartz crystal, Anal. Chim. Acta, 1981, 124, no. 1, 81-84. doi: 10.1016/S0003-2670(01)83899-X

48. T. Nomura and T. Nagamune, Internal electrolytic determination of silver in solution with a piezoelectric quartz crystal, Anal. Chim. Acta, 1983, 155, 231-234. doi: 10.1016/S0003-2670(00)85597-X

49. M. Hepel, Electrode - solution interface studied with electrochemical quartz crystal nanobalance, Interfacial Electrochemistry, Ed.: A. Wieckowski, Marcel Dekker Inc., New York - Basel, 1999, 599-630.

50. Yu.N. Mikhailovskii, Atmosfernaya korroziya metallov $i$ metody ikh zashchity (Atmospheric Corrosion of Metals and Methods of Their Protection), Metallurgiya, Moscow, 1989, p. 102 (in Russian).

51. A. Krozer and B. Kasemo, Kinetics of initial oxidation of UHV prepared Zn films, Surf. Sci., 1980, 97, no. 2, L339-L344. doi: 10.1016/0167-2584(80)90417-X

52. C.T. Kirk and E.E. Huber, The oxidation of aluminum films in low-pressure oxygen atmospheres, Surf. Sci., 1968, 9, no. 2, 217-245. doi: 10.1016/0039-6028(68)90174-X

53. M.A. Petrunin, A.P. Nazarov and Yu.N. Mikhaylovskii, Adsorption and interphase interactions in metal - silane and metal - silane - polymer systems, Prot. Met., 1993, 29, no. 2, 225-231. 
54. M.A. Petrunin, A.P. Nazarov and Yu.N. Mikhailovski, Formation mechanism and anticorrosive properties of thin siloxane films on metal surfaces, J. Electrochem. Soc., 1996, 143, no. 1, 253-256. doi: $10.1149 / 1.1836417$

55. B. Kasemo and E. Tornqvist, Quartz crystal microbalance measurements of O/Ti and CO/Ti atom ratios on very thin Ti films, Surf. Sci., 1978, 77, 209. doi: $10.1016 / 0039-$ 6028(78)90002-X

56. Characterization of Corrosion Products on Steel Surfaces, Eds.: Y. Waseda and S. Suzuki, Springer, Berlin - Heidelberg, 2006, 297 pp.

57. Testing tribocorrosion of passivating materials supporting research and industrial innovation: Handbook, Eds.: J.-P. Celis and P. Ponthiaux, European Federation of Corrosion by Maney Publishing on behalf of The Institute of Materials, Minerals \& Mining, 2012, 203 pp.

58. M. Wadsak, M. Schreiner, T. Aastrup and C. Leygraf, Combined in situ investigations of atmospheric corrosion of copper with AFM and IRAS coupled with QCM, Surf. Sci., 2000, 454-456, 246-250. doi: 10.1016/S0039-6028(00)00081-9

59. R. Schubert, B. Meagher and C.C. Chiang, Monitoring of in situ corrosion rates in a telephone switching office in Burbank, California, J. Electrochem. Soc., 1995, 142, no. 9, 3157-3164. doi: 10.1149/1.2048704

60. C. Wang, S. Zanna, I. Frateur, B. Despax, P. Raynaud, M. Mercier-Bonin and P. Marcus, BSA adsorption on a plasma-deposited silver nanocomposite film controls silver release: A QCM and XPS-based modelling, Surf. Coat. Technol., 2016, 307, 1-8. doi: $10.1016 /$ j.surfcoat.2016.07.063

61. B.B. Berkes, G. Inzelt and E. Vass, Electrochemical nanogravimetnc study of the adsorption of 4-aminoindole and the surface layer formed by electrooxidation in aqueous acid media, Electrochim. Acta., 2013, 96, 51-60. doi: 10.1016/j.electacta.2013.02.080

62. M. Itagaki, J. Kadowaki and K. Watanabe, EQCM/wall jet split-ring disk electrode study on copper dissolution in chloride aqueous solution, Anal. Sci., 2000, 16, 1049-1053. doi: 10.2116/analsci.16.1049

63. Z. Grubac, Z. Petrovic, J. Katic, M. Metikoš-Hukovic and R. Babic, The electrochemical behaviour of nanocrystalline nickel: A comparison with polycrystalline nickel under the same experimental condition, J. Electroanal. Chem., 2010, 645, 87-93. doi: 10.1016/j.jelechem.2010.04.018

64. Z. Petrovic, M. Metikoš-Hukovic and R. Babic, The electrochemical transfer reactions and the structure of the iron oxide layer electrolyte interface, Electrochim. Acta., 2012, 7, 406-413. doi: 10.1016/j.electacta.2012.05.031

65. M.A. Petrunin, L.B. Maksaeva, T.A. Yurasova, E.V. Terekhova, V.A. Kotenev, E.N. Kablov and A.Yu. Tsivadze, The directional formation and protective effect of self assembling vinyl siloxane nanolayers on copper surface, Prot. Met. Phys. Chem. Surf., 2012, 48, no. 6, 656-664. doi: 10.1134/S2070205112060081 
66. I.B. Obot and U.M. Edouk, Benzimidazole: Small planar molecule with diverse anticorrosion potentials, J. Mol. Liq., 2017, 246, 66-90. doi: 10.1016/j.molliq.2017.09.041 67. M.A. Petrunin, L.B. Maksaeva, N.A. Gladkikh, E.N. Narkevich, T.A. Yurasova, A.A. Rybkin, E.V. Terekhova, V.A. Kotenev, E.N. Kablov and A.Yu. Tsivadze, The effect of vinyl-siloxane nanolayers on the corrosion behavior of zinc, Prot. Met. Phys. Chem. Surf., 2018, 54, 795-803. doi: 10.1134/S2070205118040123

68. F. Zucchi, M. Fonsati and G. Trabanelli, Corrosion and corrosion inhibition of nickel in $\mathrm{HClO}_{4}$ solutions using the EQCM technique, J. Appl. Electrochem., 1999, 29, 347-353. doi: 10.1023/A:1003465528709 